\title{
Experimental investigation of enhanced remediation of contaminated soil using ultrasound effect
}

\author{
A.A. Adegbola ${ }^{1 *}$, A.A. Dare ${ }^{2}$, D.S. Popoola ${ }^{3}$, H.A. Saka ${ }^{3}$, A.O. Afolabi ${ }^{3}$ \\ ${ }^{1}$ Department of Mechanical Engineering, Ladoke Akintola University of Technology, Ogbomoso, Oyo State, Nigeria \\ ${ }^{2}$ Department of Mechanical Engineering, University of Ibadan, Ibadan, Nigeria \\ ${ }^{3}$ Department of Mechanical Engineering, Ladoke Akintola University of Technology, Ogbomoso, Oyo State, Nigeria \\ *Corresponding authorE-mail: saint080yinkus@yahoo.com,aaadegbola59@lautech.edu.ng
}

Copyright $($ ) 2014 A.A. Adegbola et al. This is an open access article distributed under the Creative Commons Attribution License, which permits unrestricted use, distribution, and reproduction in any medium, provided the original work is properly cited.

\begin{abstract}
In the development of an effective ground remediation method, there has been significant research focusing on the technique of enhancing soil-flushing method The soil flushing method enhanced by ultrasonic waves is a new technique that is potentially an effective method for in situ remediation of the ground contaminated by NAPL hydrocarbons. The research work investigated the effectiveness of sonication in the soil flushing method for a range of conditions involving treatment time, hydraulic gradient and the discharge velocity. The experimental investigation of the study was conducted using the inbuilt ultrasonic generator (NEE 555 timer stable multi-vibrator) and soil flushing apparatus to remove the contaminant from the soils. The test result indicated that the rate of the contaminant extraction increased considerably with increasing sonication time up to 120 seconds with $34 \%$ contaminant removed without sonication and $64.05 \%$ contaminant removed with sonication and started decreasing at the level where cavitation occurred. Increasing the sonication time also increase the contaminant removal up to the level where cavitation occurs. The effectiveness of sonication decreases with hydraulic gradient but eventually becomes constant under higher flow rates and also is highly related with the discharge velocity. Results obtained showed that sonication can enhance pollutant removal.
\end{abstract}

Keywords: Soil-Flushing Method, NAPL Hydrocarbons, Sonication, Soil Remediation, Cavitation

\section{Introduction}

Soil contamination by petroleum hydrocarbon is among the problem facing the oil and gas industry. Petroleum hydrocarbons are mostly found in urban and suburban areas due to possible leakage of gasoline, motor oils and diesel fuel from underground storage tanks. Upon completion of extraction and removal of facilities, many oil and gas lease sites may be left with varying degrees of soil contamination. Such contaminated sites pose great risks to the environment and human health. The polluted ground needs to be cleaned in order to avoid hydrocarbon contamination of ground water aquifer. There are different remediation methods such as replacement, vapor extraction, pump-and-treat and flushing methods. However a method that can be economical and also effective for a broad range of field conditions is not yet available. For development of an effective ground remediation method, there has been considerable research focusing on the technique of enhancing soil-flushing method. Soil flushing is an extraction process to remove organic compounds from contaminated soil. It removes contaminants by dissolving the liquid, sobbed or vapor phase by mobilizing contaminants existing as free product in soil pores and adsorbed to the soil [1]. Ultrasound is a sound that has frequency beyond the human hearing from $20 \mathrm{kHz}$ and above. There are data showing that ultrasonic waves are capable of removing non-aqueous phase liquid (NAPL) hydrocarbons from soils. [2] attributed the increased extraction of oil (hydrocarbons) to a decrease for water and an increase for oil in the relative phase permeability due to stress waves. [3] observed a decrease in the viscosity of polystyrene solution under sound waves. He also reported increase in oil percolation rate through porous medium. [4] developed a theoretical model to predict removal of small particle and fines in porous media. [5] stated that ultrasound can reduce the viscosity of high polymeric liquid by up to $27 \%$. It was reported that ultrasonic excitation can suspend fine particle to which the contaminants are strongly absorbed [6]. Also, [7],[8] presented that ultrasonic waves can increase not only the mobility of NAPL ganglia but the porosity of the soil as 
well, resulting in a decrease in viscosity and buoyant pressure. [9] stated that sonication can enhance pollutant removal considerably and that the degree of enhancement depends on a number of factors such as sonication power, water flow rate and soil type. [10] reported a $30 \%$ increase in contaminant extraction due to acoustic excitation. Another study by [11] reported a 6-26\% improvement in contaminant extraction. Hence the soil-flushing method enhanced by ultrasonic waves is a new technique that potentially can become an efficient method for in situ remediation of the ground contaminated by NAPL hydrocarbons. The objectives of this research work is to investigate the effectiveness of ultrasound on the soil flushing remediation technique for a range of condition involving soil type,sonication time and flushing rate.

\section{Laboratory investigation}

The experiment was carried out at the fluid laboratory, Mechanical Engineering department of Ladoke Akintola University of Technology, Ogbomoso, and Oyo state. The experimental set up was designed for both soil flushing treatment and ultrasonic treatment. The system consists of three parts namely ultrasonic processor, test chamber and soil flushing apparatus. The ultrasonic processor consists of generator, a converter and acoustic horn. The test chamber was made of aluminium cylinder. The soil flushing apparatus consists of water reservoir, connecting pipe and water tap.

\subsection{Experimental setup description}

The test chamber was made of aluminum cylinder with $15 \mathrm{~cm}$ diameter and height of $20 \mathrm{~cm}$. The water inlet pipe coming from the de-aired water in the reservoir was connected to the test chamber at the bottom to enable the flushing out of contaminant from the soil sample. And the outlet pipe led to a measuring beaker to collect the effluent. The water reservoir containing the de-aired water was placed at a considerable height to generate the desired hydraulic gradient. And finally mounted on the test chamber was the acoustic horn which introduced the effect of vibration.

\subsection{The ultrasonic processor working principle}

The power supply to the generator was converted from a conventional $50 \mathrm{~Hz} \mathrm{AC}$ at $220 \mathrm{~V}$ to $20 \mathrm{kHz}$ electrical energy at approximately $1000 \mathrm{~V}$. The high frequency electrical energy was fed to the converter to transform the high frequency electrical energy to mechanical vibration and the converter vibrated at $20 \mathrm{kHz}$. The acoustic horn containing the flat tip then amplified the longitudinal vibration of the converter which was fed directly into the test Chamber.

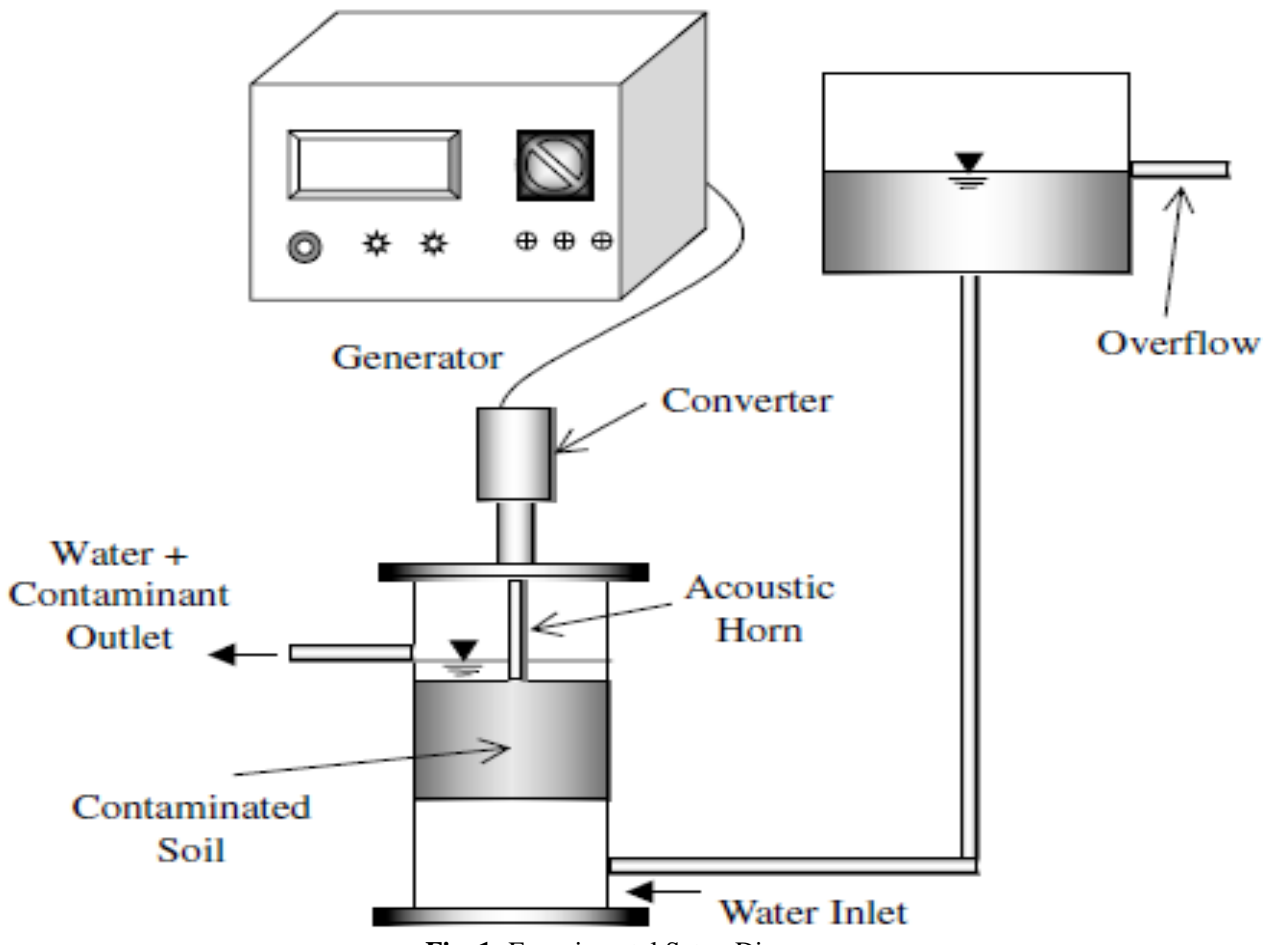

Fig. 1: Experimental Setup Diagram 


\subsection{Soil sample and contaminant}

The soil sample made available was sandy soil of grain sizes between $0.45 \mathrm{~mm}-1.10 \mathrm{~mm}$. The contaminant chosen for this research work is Diesel which is produced from the fractional distillation of crude oil between $200^{\circ} \mathrm{c}$ and $350^{\circ} \mathrm{c}$ at atmospheric pressure thereby resulting in a mixture of carbon chains that typically contain between 8 and 12 carbon atmosphere molecule. It has a density of $0.918 \mathrm{~g} / \mathrm{mol}$ at $20^{\circ} \mathrm{c}$ and viscosity of $65 \mathrm{cst}$ at $20^{\circ} \mathrm{c}$.

\subsection{Experimental procedure}

The research work was divided into two sections. The first part was related to ultrasonic enhancement of soil flushing remediation technique. In this part, the experiment was designed using an inbuilt ultrasonic generator and soil flushing mechanism combined together to treat the soil contaminated with NAPL oil. The second part was soil flushing remediation technique without ultrasonic waves. There was weighing of the soil sample to know the volume it will occupy in the test chamber. Measurement of the amount of contaminant to be used to know the initial volume of the contaminant was taken.Pre-weighed soil sample was thoroughly mixed with the contaminant in a container. The sample (soil + contaminant) was carefully placed into the test chamber. The mixture was then saturated with water, keeping the water level maintained at the top of the soil specimen. The ultrasonic processor was switched on to generate ultrasound effects at a frequency of $20 \mathrm{kHz}$. For a given period of time (t), the clean water from the reservoir was allowed to flow upward through the soil contaminant mixture under a specified hydraulic gradient. Effluent (water + oil) flowed out of the test chamber through the outlet pipe into a measuring cylinder. The effluent in the measuring cylinder was allowed to stand overnight for gravitational segregation of oil from water. The volume of the separated oil and water was then measured.

\section{Results and discussions}

Table $1-3$ below show the results of the experiment carried out on soil flushing and soil flushing enhanced with ultrasound. From the graphs, it is shown that numerous factors may influence the percentage of contaminant removal. Major factors investigated were sonication time, hydraulic gradient and discharge velocity. The effect of these factors on contaminant removal was investigated on the sandy soil specimens that were prepared.

Table 1: Percentage of Contaminant Removed With Respect To Time

\begin{tabular}{ccc}
\hline Treatment Time (seconds) & Contaminant removed Without sonication $(\%)$ & Contaminant removed With sonication $(\%)$ \\
\hline 20 & 23.55 & 53.55 \\
40 & 26.42 & 56.42 \\
60 & 29.05 & 59.03 \\
80 & 31.28 & 61.36 \\
100 & 32.80 & 62.83 \\
120 & 34.00 & 64.05 \\
140 & 33.98 & 63.02 \\
160 & 33.62 & 62.98 \\
180 & 32.88 & 62.63 \\
200 & 32.66 & 62.58 \\
\end{tabular}

Table 2: Of Contaminant Removed With Respect To Hydraulic Gradient

\begin{tabular}{ccc} 
& Table 2: Of Contaminant Removed With Respect To Hydraulic Gradient \\
\hline Hydraulic gradient $(\mathrm{cm})$ & Contaminant removed without sonication $(\%)$ & Contaminant removed with sonication $(\%)$ \\
\hline 5 & 38.30 & 68.00 \\
10 & 35.00 & 65.50 \\
15 & 32.70 & 62.72 \\
20 & 30.50 & 60.53 \\
25 & 29.06 & 59.04 \\
30 & 28.55 & 58.54 \\
35 & 28.04 & 58.03 \\
40 & 27.83 & 57.83 \\
45 & 27.66 & 57.68 \\
50 & 26.81 & 56.87 \\
\hline
\end{tabular}

Table 3: Table of Contaminant Removed With Respect To Discharge Velocity

\begin{tabular}{ccc} 
& \multicolumn{2}{c}{ Table 3: Table of Contaminant Removed With Respect To Discharge Velocity } \\
\hline Discharge $(/ \mathrm{s})$ & Contaminant removed without ultrasound $(\%)$ & Contaminant removed with ultrasound (\%) \\
\hline 1.5 & 36.35 & 66.33 \\
2.5 & 28.85 & 58.85 \\
3.5 & 26.00 & 56.02 \\
4.5 & 25.49 & 55.48 \\
5.5 & 25.47 & 55.48 \\
\hline
\end{tabular}


Fig. 1 presents the graphical relations between treatment time and percentage of contaminant removed for the experimental investigation. The effect of treatment time on contaminant removal was carried out at hydraulic gradient of $20 \mathrm{~cm}$. It was observed that at a constant hydraulic gradient, the percentage of contaminant removed increases with the treatment time for both ultrasonic enhanced soil flushing and the soil flushing processes to a maximum around 120 seconds of treatment then decreases with the percentage of contaminant removed. It is obvious that if the treatment time increases further, the reduction will increase further. The reduction in contaminant removal after 120 seconds is due to the effect of cavitation. Cavitation is the formation and then immediate implosion of cavities in a liquid i.e. bubbles that are the consequence of forces acting upon the liquid. When cavitation occurs, the sound pressure level at a distance drops because cavitation takes power away from the field. The graph also shows the effectiveness of ultrasound in contaminant removal in a porous medium as it increases the percentage of contaminant removed all other factors being kept constant.

Fig. 2 presents the graphical relations between hydraulic gradient and percent contaminant removed for the experimental investigation carried out with hydraulic gradients interval of $5 \mathrm{~cm}$ with a fixed treatment of $60 \mathrm{~seconds}$. At low hydraulic gradient, there is higher percentage of contaminant removed. The percentage of contaminant removal decreases with increasing hydraulic gradient. The explanation is that, increasing the hydraulic gradient will increase discharge velocity and flow rate, therefore reducing the time for the flushing water to interact with the soil/contaminant system. For low hydraulic gradient, the water has longer time to interact with the system, which makes it more efficient to remove the contaminant than fast flushing under high hydraulic gradient.

Similarly, Fig. 3 shows the percent contaminant removed vs. discharge velocity in the experimental investigation. It can be deduced from the relationship between the hydraulic gradient and discharge velocity which is linearly proportional i.e. the higher the hydraulic gradient the higher the discharge velocity. At lower discharge velocity, more contaminant was removed due to low flushing time for long interaction between the flushing water and the soil/contaminant system.

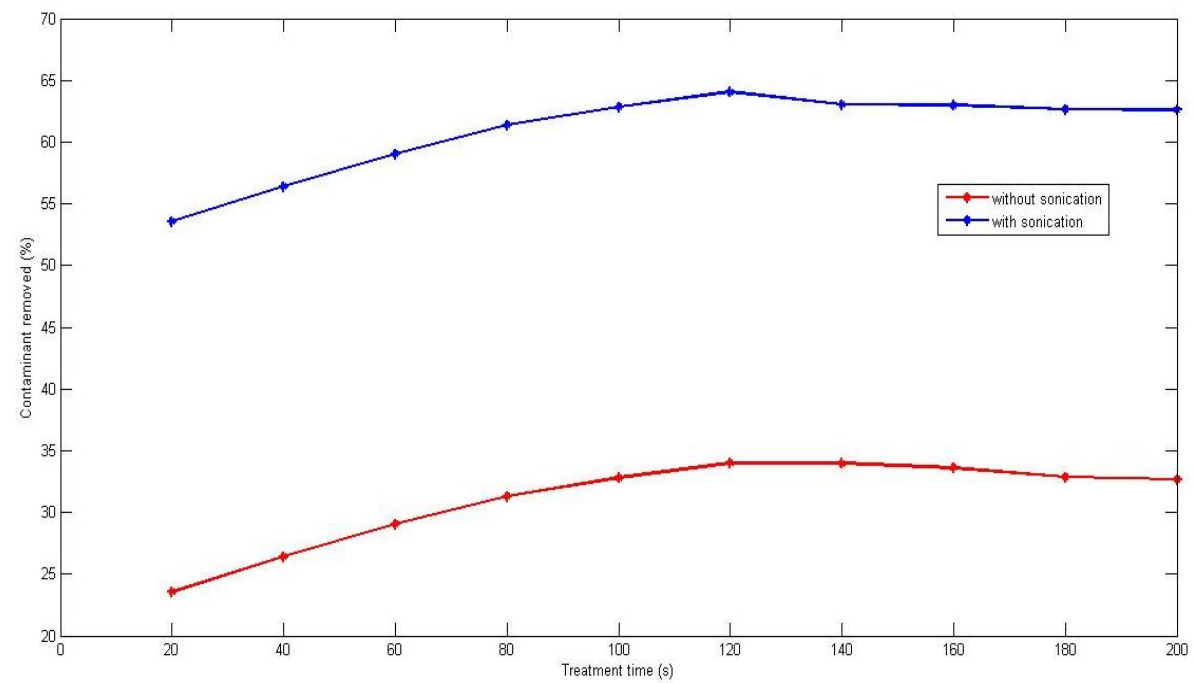

Fig. 1: Relationship between Treatment Time and Percentage Contaminant Removed

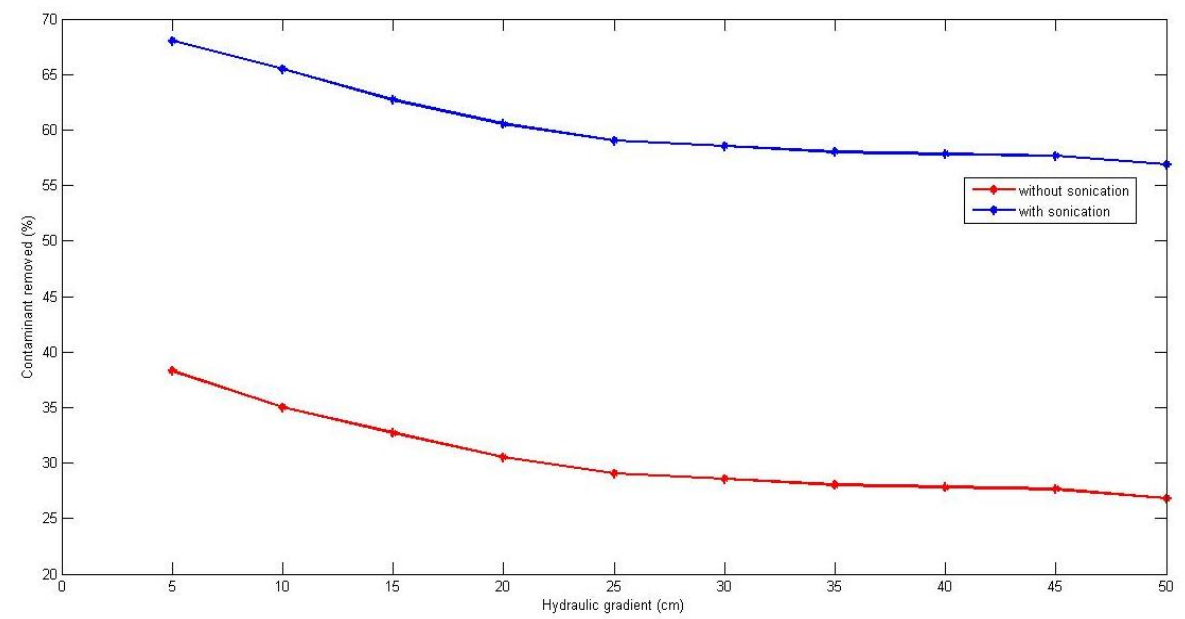

Fig. 2: Relationship between Hydraulic Gradient and Percentage Contaminant Removed 


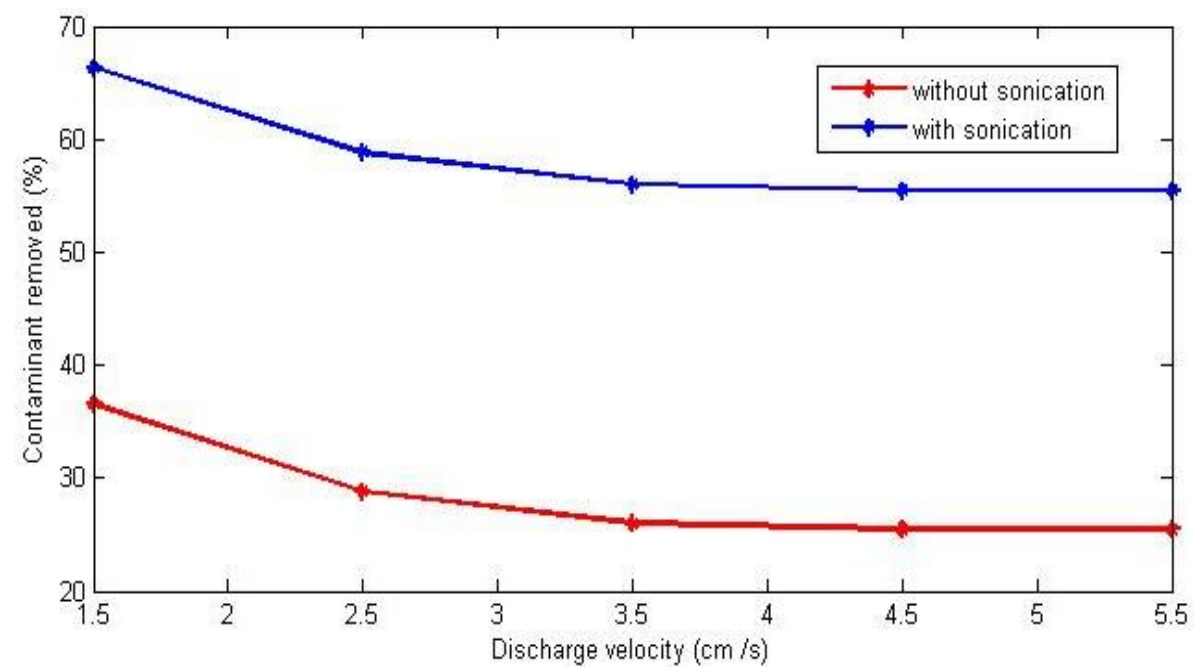

Fig. 3: Relationship between Discharge Velocity and Percentage Contaminant Removed

\section{Conclusions}

This study investigated the effectiveness of ultrasound enhancement in the soil-flushing method. The results show that sonication can enhance contaminant removal considerably. The results indicated the following:

i) Sonication increases the efficiency of soil flushing in soil remediation. It enhances contaminant removal considerably and the degree of enhancement depends on a number of factors.

ii) Increasing the sonication time increase the contaminant removal up to the level where cavitation occurs.

iii) The effectiveness of sonication decreases with hydraulic gradient but eventually becomes constant under higher flow rates and is highly related with the discharge velocity.

\section{Acknowledgements}

The authors wish to thank Dr. J.A. Ojo and Engr. K.O. Oladosu for their assistance during this work.

\section{References}

[1] Hyman, M. Norman, R.R. and Reidy, E.SGroundwater and soil remediation - process design and cost.Pg 176-189, 2001.

[2] M.L. Surguchev and E.M. Simkin Advanced vibroseismic action on an oil-bearing bed, Journal of Engineering Physics and Thermophysics 64(4) Pg 355-359, 1991

[3] Johnston, H. K., II. "Polymer Viscosity Control by the Use of Ultrasonics." Chemical Engineering Progress Symposium Series 67, Pg 39-45, 2004.

[4] Poesio, P. and Ooms, G "Formation and Ultrasonic Removal of Fouling Particle Structures in a Natural Porous Material." Journal of Petroleum Science and Engineering 45(3-4): Pg159-178, 2004.

[5] Gadiev, S. M. "Use of Vibrations in Oil Production (Ispol'zovaniyeVibratsii DobycheNefti)." Moscow, Nedra Press, 2003.

[6] Cleveland, T.G, Garg, S. Field demonstration of acoustically enhanced soil washing system for in-situ treatment of low-permeability soils, in:, Pg 519-520,2003.

[7] Reddi, L.N., Wu, H. Mechanism involved in vibratory destabilization of NAPL ganglia in sands, Journal of Environmental Engineering, ASCE 122 (12). Pg 1115-1119, 1995.

[8] Reddi, L.N., S. Challa Vibratory mobilization of immiscible liquid ganglia in sands, Journal of Environmental Engineering, ASCE 120 (5). Pg 1170-1190,1994.

[9] Kim, Y.U., and Wang, M.CEffect of ultrasound on oil removal from soils. Ultrasonics 41:Pg539-542, 2003.

[10] T.V. Ellen C.J.E. Lansink, J.E. Sandker Acoustic soil remediation:introduction of a new concept, Contaminated soil 95, Pg 1193-1194, 1995.

[11] J.L. Iovenitti, T.M., Rynne, J.W. Spencer Jr., Acoustically enhanced remediation of contaminated soil and ground water, in:Proceedings of Opportunity 95-Environmental Technology through Small business, Morgantown, West Virginia, 1995. 